\title{
Development of an Anger camera in lanthanum bromide for gamma-ray space astronomy in the $\mathrm{MeV}$ range
}

\author{
Vincent Tatischeff*, J. Kiener, G. Sedes, C. Hamadache, N. Karkour, D. Linget \\ CSNSM, IN2P3-CNRS and Univ Paris-Sud, 91405 Orsay Campus, France \\ E-mail: Vincent.Tatischeffecsnsm.in2p3.fr
}

\section{A. T. Astorino, D. C. Bardalez Gagliuffi}

CSNSM, France, \& MIT, 77 Massachusetts Avenue, Cambridge, MA, USA

\section{S. Blin, P. Barrillon}

LAL, IN2P3-CNRS and Univ Paris-Sud, 91898 Orsay cedex, France

\begin{abstract}
Lanthanum bromide is a very promising scintillator material for the next generation of $\gamma$-ray telescopes. We present in this paper first $\gamma$-ray imaging results obtained by coupling a $\mathrm{LaBr}_{3}$ crystal with a position-sensitive $8 \times 8$ multianode photomultiplier tube to form a simple Anger camera module. The readout of the 64 signals is carried out with the most recent evolution of the MultiAnode ReadOut Chip (MAROC) which was initially designed for the luminometer of the ATLAS detector. Measured charge distributions are compared with detailed GEANT4 simulations that include the tracking of the optical photons produced in the scintillation crystal. The depth of interaction (d.o.i.) of 662-keV $\gamma$-rays inside the crystal is derived from the charge distributions using an artificial neural network. We obtain for an irradiation at detector centre a mean standard deviation of the d.o.i. of $1.69 \mathrm{~mm}$. Such a position-sensitive $\gamma$-ray detector can form an innovative building block for a future space calorimeter
\end{abstract}

8th INTEGRAL Workshop "The Restless Gamma-ray Universe"

September 27-30, 2010

Dublin Castle, Dublin, Ireland

\footnotetext{
* Speaker.
} 


\section{Introduction}

The recently developed cerium-doped lanthanum bromide $\left(\mathrm{LaBr}_{3}: \mathrm{Ce}\right)$ inorganic scintillator is a very promising material for $\gamma$-ray astronomy. It has a high stopping power - we measured its intrinsic efficiency to be 1.3 times higher than that of $\mathrm{NaI}$ at $1 \mathrm{MeV}$ - and can be fabricated in large volumes, thus offering the possibility of high detection efficiency for medium-to-high-energy $\gamma$-rays. Its energy resolution, 2.9\% and 1.6\% FWHM at 662 and $2615 \mathrm{keV}$, respectively (see [1]), is comparable to the one of semiconductor detectors operating at room temperature (such as CZT). With a fluorescent 1/e decay time $\tau_{d}=16 \mathrm{~ns}$ and a measured coincidence resolving time lower than $200 \mathrm{ps}$ [1], it is much faster than other scintillating materials (e.g., $\tau_{d}=300 \mathrm{~ns}$ for BGO), thus offering the opportunity of fast coincidence timing for efficient background rejection. It has a satisfactory radiation tolerance to high-energy proton irradiation with fluences typical of those in the interplanetary space $[2,3]$ and also has sufficient radiation hardness when exposed to a high flux of $\mathrm{MeV} \gamma$-rays [4]. For all of these reasons, $\mathrm{LaBr}_{3}$ is currently under study for various space missions. Thus, it has been chosen for the Mercury Gamma and Neutron Spectrometer (MGNS) of the BepiColombo mission, scheduled to be launched in 2014 [5]. This scintillator will also be used in the high-energy instrument XGRE of the TARANIS mission, which is a microsatellite of the Centre National d'Etudes Spatiales (CNES) also to be launched in 2014 [6]. Furthermore, the use of $750 \mathrm{~kg}$ of $\mathrm{LaBr}_{3}$ scintillator crystals has been proposed for the main instrument of the Gamma-Ray Imaging, Polarimetry and Spectroscopy (GRIPS) mission (see [7] and Diehl et al. in these proceedings).

One of the most critical aspects of this scintillator for a future use in $\gamma$-ray astronomy is the 3D position resolution achievable in large-volume $\mathrm{LaBr}_{3}$ crystals. Future $\gamma$-ray telescopes operating in the $\mathrm{MeV}$ range could take advantage of such advanced position-sensitive detectors for better background rejection, Compton imaging and polarization measurement. To study this aspect, following a concept proposed by McConnell et al. [8] for the CASTER mission project, we have coupled a $\mathrm{LaBr}_{3}$ crystal with a position-sensitive multianode photomultiplier tube (MAPMT) to form an Anger camera module. The principle of the $\gamma$-ray hit localization with such a device is illustrated in Figure 1. In addition to the localization of the interaction site in the $X-Y$ sensor plane, the distribution of scintillation signals within this plane also provides a measure of the $Z$-coordinate, i.e. the depth of interaction (d.o.i.) inside the crystal. Indeed, the distribution is expected to be narrower for interactions occurring close to the photocathode. We present here the first results obtained at Orsay with this detector prototype.

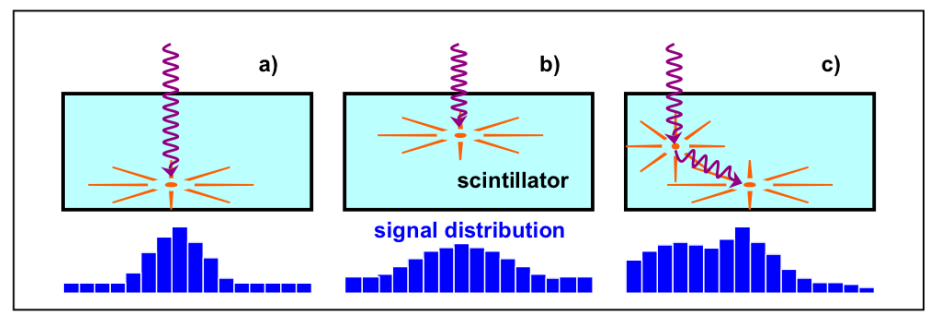

Figure 1: Gamma-ray hit localization in a scintillator coupled to a MAPMT: distributions of scintillation signals for $(a)(b)$ two depths of $\gamma$-ray photoelectric interaction and $(c)$ a Compton plus photoelectric event. 


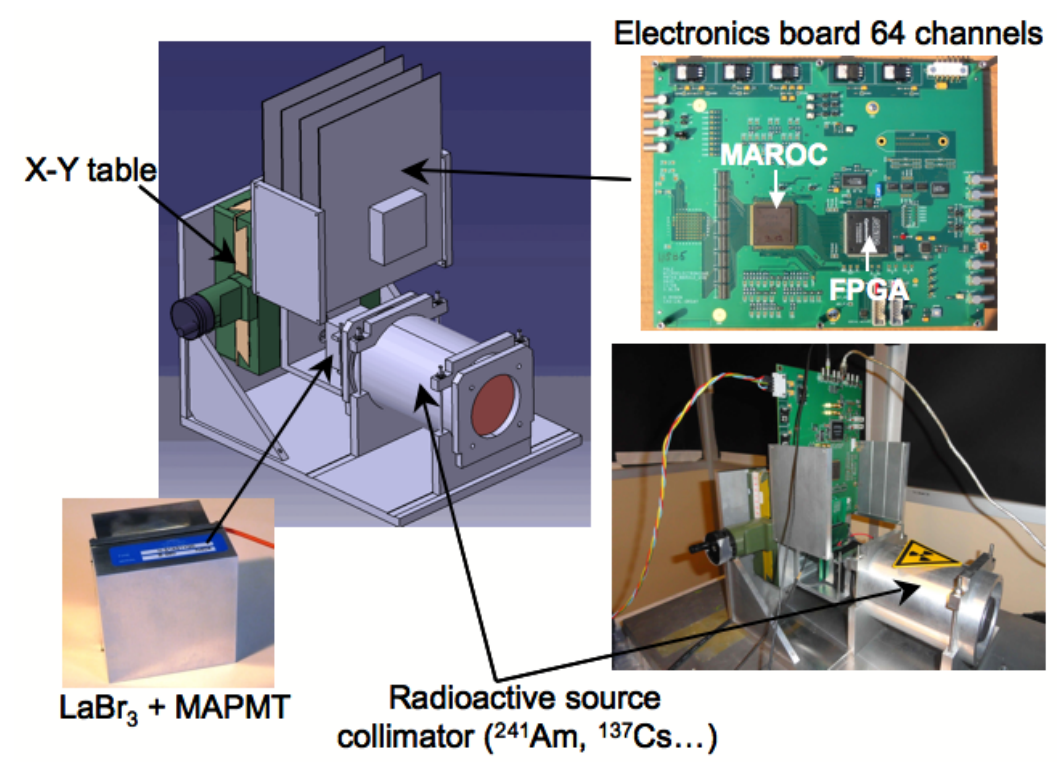

Figure 2: Test bench dedicated to the position resolution measurements.

\section{Experimental set-up}

The test bench installed at CSNSM for the position resolution measurements in shown in Figure 2. The detector is made of a $\mathrm{LaBr}_{3}$ :Ce crystal grown by Saint Gobain Crystals and Detectors of $50 \times 50 \mathrm{~mm}^{2}$ detection area and $20 \mathrm{~mm}$ thickness coupled to a Hamamatsu H8500 Flat Panel $8 \times 8$ MAPMT. The entrance face of the $\mathrm{LaBr}_{3}$ crystal was grinded and covered with a white diffusive reflector in order to maximize the scintillation light output, whereas the side faces were polished and black painted to avoid position distortions due to the light reflections. The detector is mounted on an X-Y table in front of a collimator made of lead and tantalum, inside which various radioactive sources can be placed. The readout of the signals is carried out with the most recent evolution of the ASIC MAROC (MultiAnode ReadOut Chip), which was designed at LAL for the luminometer of the ATLAS detector [9]. It is a 64-channel input circuit of $4 \times 4 \mathrm{~mm}^{2}$ area based on AMS Si-Ge 0.35 $\mu \mathrm{m}$ technology, supplying 64 trigger outputs, a multiplexed charge output, as well as 64 digitized charge outputs furnished by a 12-bit Wilkinson Analog-to-Digital Converter. We use in this work the electronics board (Fig. 2) and associated LabVIEW software developed at LAL for the chip characterization. The electronic chain was enhanced at CSNSM by transforming the MAROC test board into a 64 channel data acquisition system with the creation of a digitally-controlled trackand-hold signal from the trigger output, so as to obtain an auto-triggered system of charge signal acquisition. The system was fully characterized and found to be compatible with the requirements of the measurements. The experimental set-up is now complete and first position measurements have been performed.

As an example, Figure 3 shows two charge distributions measured with a collimated ${ }^{137} \mathrm{Cs}$ source producing a $662-\mathrm{keV} \gamma$-ray beam pointing at the detector centre. We see that after correction of the non-uniformity of the gain of the PMT anodes, for which we used the response matrix provided by Hamamatsu Photonics, the distribution is well symmetric around the centre. The 

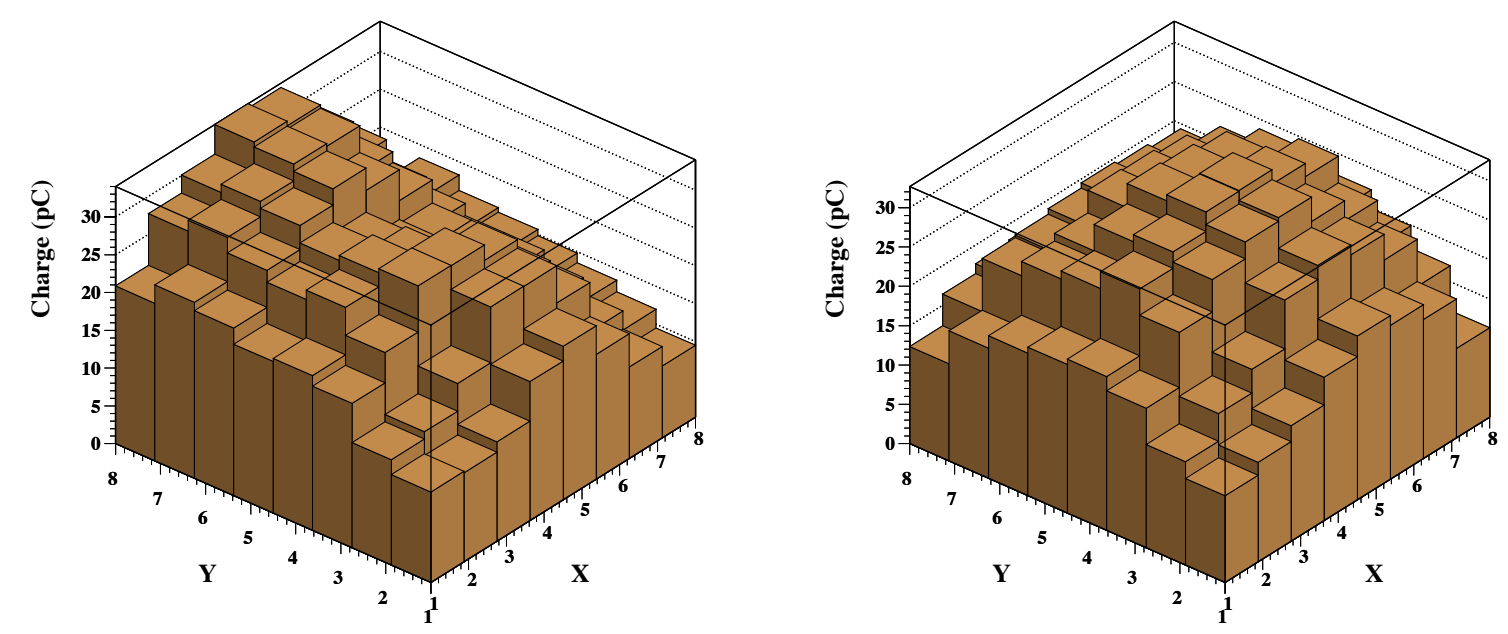

Figure 3: Charge distributions for fully-absorbed $662-\mathrm{keV} \gamma$-rays measured with a collimated ${ }^{137} \mathrm{Cs}$ source pointing at the detector centre, before (left panel) and after (right panel) correction of the non-uniformity of the PMT anodes.

measured total mean charge produced by $662-\mathrm{keV} \gamma$-rays is $Q=1455 \mathrm{pC}$. With a mean MAPMT amplification of $1.2 \times 10^{6}$ for a power supply of $1 \mathrm{kV}$ (as given by the manufacturer) the measured charge corresponds to a photoelectron yield of $11.4 \mathrm{keV}^{-1}$.

\section{GEANT4 simulations of the scintillation signal distributions}

The measurements are compared with detailed GEANT4 simulations that include the tracking of the optical photons produced in the scintillator. The behaviour of the optical photons at surface boundary is treated with the so-called UNIFIED model [10], which allows us to correctly take into account the effects of the detector surface finish and coating (see above). We use reflectivities of $97 \%$ and $4 \%$ for the white diffusive reflector and black paint absorber, respectively, and took for the poorly-known bulk absorption length of scintillation photons in $\mathrm{LaBr}_{3} \lambda_{B}=20 \mathrm{~cm}$, based on Ref. [3]. The $\mathrm{LaBr}_{3}$ :Ce scintillation light yield amounts to 63 photons $\mathrm{keV}^{-1}$ [1]. The photocathode is as present treated as a simple dielectric-metal interface with a constant absorption coefficient of $60 \%$ and photoelectron efficiency of $50 \%$.

We compare in the left and middle panels of Figure 4 two charge distributions obtained by simulating two events with very different locations of the first Compton scattering interaction: one occurring at $Z_{1}=1.7 \mathrm{~mm}$ from the detector entrance surface and the other occurring close to the photocathode, at $Z_{1}=18.3 \mathrm{~mm}$. This illustrates how sensitive can be the distribution of scintillation signals to the depth of the first interaction. The right panel of this figure shows the mean distribution reconstructed from all simulated events. We see that the measured distribution (Figure 3) is reasonably well reproduced. In particular, the simulation returns a total mean charge of $1431 \mathrm{pC}$ (or a photoelectron yield of $11.2 \mathrm{keV}^{-1}$ ), in excellent agreement with the measured value. With the adopted value of $\lambda_{B}, 29 \%$ of the generated optical photons are self-absorbed in the scintillation crystal, $34 \%$ are lost at surface boundaries, the other photons (37\%) being absorbed at the photocathode. The simulated distribution is however slightly narrower than the measured one. 

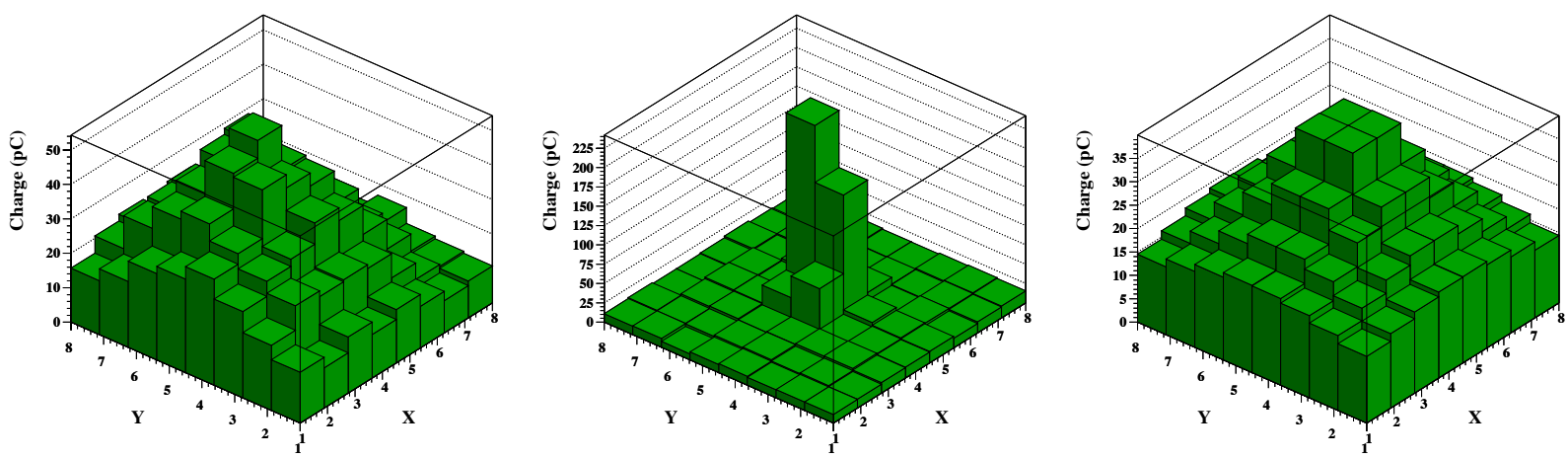

Figure 4: Simulated charge distributions for fully-absorbed $662-\mathrm{keV} \gamma$-ray irradiation at detector center. Left panel: an event with first hit d.o.i. $Z_{1}=1.7 \mathrm{~mm}$ and a deposited energy in the first interaction $E_{1}=581 \mathrm{keV}$; middle panel: $Z_{1}=18.3 \mathrm{~mm}$ and $E_{1}=528 \mathrm{keV}$; right panel: all simulated events.

It is possible that a better treatment of the photocathode in the simulation [11] would improve the agreement with the data.

To solve the inverse problem consisting in the estimate of the d.o.i. from the measured charge distributions, we found that the best method is to use an artificial neural network (ANN), for which we adopted the JETNET 3.0 package [12]. The best results were obtained with a feed-forward multilayer perceptron comprising two hidden layers of 35 nodes each, with back-propagation Langevin updating rule. We use as inputs of the ANN the 64 channels of the distribution. The network was trained with a large number of simulated data for various detector irradiation positions. We obtained for an irradiation at detector centre by $662-\mathrm{keV} \gamma$-rays a mean standard deviation of the d.o.i. of $1.69 \mathrm{~mm}$ (see left panel of Figure 5), with even better d.o.i. resolution in most of the crystal volume (right panel). This result is now being further compared to experimental data.

We wish to emphasize that the use of an ANN to store the position response function of a continuous Anger-camera-like module provides a convenient way to measure in real time the $\gamma$-ray interaction location in the detector. It is indeed a simple operation to derive the $3 \mathrm{D}$ position from the measured charge distribution, once known the weight matrix of a previously trained ANN.
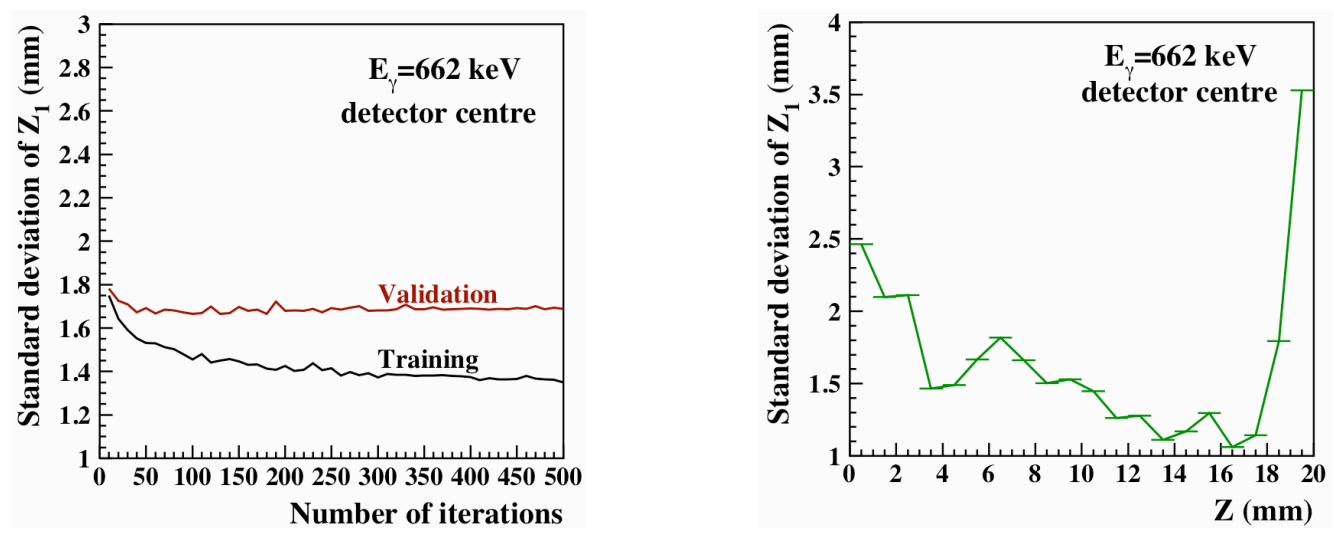

Figure 5: Left panel: mean standard deviation of $Z_{1}$ returned by the ANN as a function of the number of iterations in the training process; right panel: standard deviation of $Z_{1}$ as a function of the $Z$-position inside the $\mathrm{LaBr}_{3}$ crystal, at iteration 500 . 


\section{Conclusion}

The first results obtained in this research and development project show that continuous Angercamera-like modules in $\mathrm{LaBr}_{3}$ can form innovative building blocks for a future $\gamma$-ray calorimeter. Such an instrument could be combined with a tracker made of 3D position sensitive double-sided silicon stripped detectors to build a Compton telescope much more sensitive in the $\mathrm{MeV} \gamma$-ray energy range than the previously flown missions.

\section{References}

[1] Saint-Gobain Technical Note BrilLanCe Scintillators: Performance Summary (January 2009), http://www.detectors.saint-gobain.com/

[2] A. Owens et al., Nucl. Instr. and Meth. in Phys. Res. A572 (2007) 785

[3] W. Drozdowski et al., IEEE Trans. Nucl. Sci. 54 (2007) 736

[4] W. Drozdowski et al., IEEE Trans. Nucl. Sci. 54 (2007) 1387

[5] I. G. Mitrofanov et al., Planetary Space Sci.58 (2010) 116

[6] F. Lefeuvre et al., Space Sci. Rev. 137 (2008) 301 ; see also http://www.apc.univ-paris7.fr/APC_CS/en/experience/taranis/presentation

[7] J. Greiner et al., Experimental Astronomy 23 (2009) 91

[8] M. L. McConnell et al., New Ast. Rev. 50 (2006) 63

[9] S. Blin, P. Barrillon, et al., Technical Note MAROC3 Datasheet (August 2010), http://omega.in2p3.fr/

[10] A. Levin \& C. Moisan, TRIUMF Preprint TRI-PP-96-64, Oct. 1996

[11] D. Motta \& S. Schonert, Nucl. Instr. and Meth. A539 (2005) 217

[12] C. Peterson et al., Comput. Phys. Commun. 81 (1994) 185 